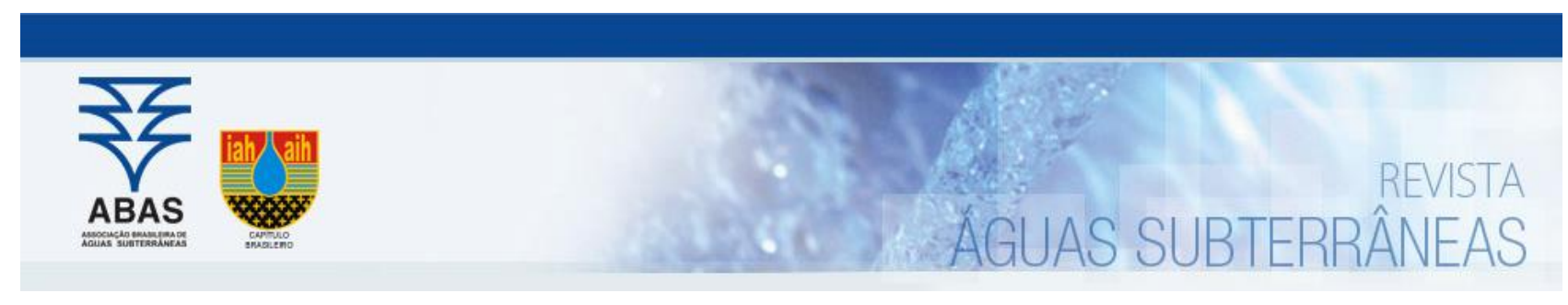

Artigos

\title{
Uma solução para a equação de Richards em perfis de solos em camadas com a abordagem de domínio único
}

\section{A solution for the Richards equation in layered soil profiles with a single domain approach}

\author{
Diego Lopes'; Diego Estumano1; Emanuel Macêdo1; João Quaresma1 ${ }^{\square}$ \\ 1Universidade Federal do Pará (UFPA), Belém, PA
}

凶diegosousa.eng@gmail.com, dcestumano@ufpa.br, enegrao@ufpa.br, quaresma@ufpa.br

\author{
Palavras-chave: \\ Equação de Richards: \\ Enfoque de domínio único; \\ Método das linhas.
}

\begin{abstract}
Resumo
O presente artigo tem como objetivo desenvolver uma solução para equação de Richards para o escoamento unidimensional de água através de perfis de solo em camadas, usando uma abordagem em domínio único. Foi proposto uma formulação matemática em que uma função de transição é inserida na modelagem para suavizar as descontinuidades presentes nas interfaces. O Método das Linhas (MOL), juntamente com um esquema de volumes finitos, é usado para resolver o problema. Uma comparação é realizada entre os resultados obtidos e aqueles da literatura para verificação do modelo. Para todos os casos estudados, efetuou-se uma análise de convergência e experimentos numéricos foram realizados para analisar as influências dos parâmetros físicos Ks e $\alpha$. Resultados foram obtidos em termos de teor de umidade $(\theta)$ e convertidos em termos de pressão hidráulica $(\psi)$, apresentando boa concordância com os valores da literatura, mostrando que a abordagem do problema em domínio único pode lidar com a descontinuidade presente na interface, e que a função de transição sugerida é um caminho viável para a solução da equação de Richards.
\end{abstract}

Keywords:

Richards equation;

Single domain approach;

Method of lines.

Revisão por pares.

Recebido em: 16/03/2021.

Aprovado em: 31/08/2021.

\begin{abstract}
This article aims to develop a Richards equation solution for one-dimensional water flow through layered soil profiles, using a single domain approach. A mathematical formulation was proposed in which a transition function is inserted in the modeling to smooth out the discontinuities present at the interfaces. The Method of Lines (MOL), together with a finite volume scheme, is used to solve it. A comparison is made between the results obtained and those in the literature to verify the model. For all the cases studied, a convergence analysis was carried out, and numerical experiments were performed to analyze the influences of the physical parameters Ks and $\alpha$. Results were obtained in terms of moisture content $(\theta)$ and converted in hydraulic pressure $(\psi)$, showing good agreement with the literature values. Such results showed that the problem in a single domain approach could deal with the discontinuity present at the interfaces; this way, the suggested transition function is viable to solve the Richards equation.
\end{abstract}

DOI: http://doi.org/10.14295/ras.v35i2.30022

\section{INTRODUÇÃO}

Os fenômenos de infiltração e movimentação da água no solo são tópicos essenciais para o estudo da hidrologia e do ciclo de ecossistemas vegetais. Em tais eventos, a capacidade de inferir a umidade do solo e sua interação com a vegetação tem excelentes práticas em hidrologia, ciência do clima, engenharia, agricultura e gestão de ecossistemas (WENDLAND; PIZARRO, 2010; BRODBRIDGE et al., 2017; KANZARI; MARIEM, 2017).

A distribuição da umidade no solo é o movimento da água infiltrada na zona não saturada do solo, impulsionada por entradas de água via infiltração superficial, como chuva, degelo, irrigação, e fluxos causados pela evaporação e retirada de água pelas raízes (LAIO et al., 2001; WENDLAND; PIZARRO, 2010; KANZARI; MARIEM, 2017; ZHU et al., 2018). Geralmente, o modelo usado para dinâmica de água no solo é descrito pela equação de Richards (RICHARDS, 1931), a qual combina a lei de Darcy estendida a solos insaturados com conservação de massa, como visto em (PHILIPP, 1969; HILSS et al., 1989; CELIA et al., 1990).

As soluções desta equação por sua vez são difíceis de serem obtidas, sejam elas analíticas ou numéricas, isso se dá pelas relações não lineares que ligam a umidade do solo à condutividade hidráulica do solo e ao potencial matricial (HILLEL, 1998; KANZARI; MARIEM, 2017). Modelos numéricos disponíveis para resolver a tal equação são diferenciados pelas formas da mesma, além das diferentes técnicas de discretização e métodos numéricos aplicado às suas soluções. As diferentes abordagens podem influenciar significativamente a eficiência computacional, a precisão e o comportamento numérico das soluções (ZHA et al., 2013).

Os solos por sua vez são inerentemente heterogêneos em diversas escalas, geralmente os perfis de solos mostram características em camadas, nas quais possuem grandes influências nos fenômenos de infiltração, redistribuição da umidade de água no solo e recarga das águas subterrâneas. As proprie- 
dades hidráulicas, como a condutividade hidráulica não saturada, podem variar de magnitude em diferentes camadas, enquanto a umidade do solo pode ser descontínua nas interfaces. Esses fatores representam desafios para a solução numérica da equação de Richards (ZHA et al., 2019).

A forma $\theta$ da equação de Richards, em que $\theta$ é o conteúdo de água, geralmente não é usada para solos em camadas, dado a existência de uma descontinuidade no valor de $\theta$ na interface. Hilss et al. (1989) foram os primeiros a desenvolverem um modelo em termos de $\theta$ para infiltração em um perfil de solo em camadas. 0 modelo foi desenvolvido para um escoamento unidimensional e constante, através de um perfil de solo com cinco camadas em condições muito secas, usando técnicas de diferenças finitas, usadas para resolver os modelos na forma de pressão $\psi$ e $\theta$ (MATTHEWS et al., 2004). E, para satisfazer as condições de contorno na interface, um sistema de malha foi construído em que a interface está localizada entre dois nós de diferenças finitas, e um salto foi especificado entre dois nós próximos na interface para contabilizar a descontinuidade em $\theta$. Isso permitiu uma solução direta para o conteúdo de água na interface $\left(\theta_{c}\right)$ (HILSS et al., 1989; MATTHEWS et al., 2004).

Romano et al. (1998) usaram a forma $\psi$ da equação de Richards, com a aplicação da solução de Hilss et al. (1989), e melhoraram o esquema numérico introduzindo nós fictícios na interface entre os domínios em ambos os lados das fronteiras, resultando em expressões idênticas na interface como visto em (HILSS et al., 1989) para $\left(\psi_{c}\right)$. Contudo, o novo esquema exigia uma solução iterativa para determinar os valores de $\psi$ nos nós fictícios. Além disso, mostraram que a abordagem em (HILSS et al., 1989) pode produzir um erro na interface de até $4 \%$ quando comparado à solução analítica de Srivastava e Yeh (1991).

Matthews et al. (2004) resolveram a equação de Richards usando o método das linhas (do inglês Method of Lines - MOL), e a descontinuidade de umidade é considerada por um algoritmo explícito, e logo depois melhoraram a estabilidade do modelo usando um algoritmo implícito para contabilizar a descontinuidade da umidade do solo, em que também usaram nós fictícios na interface, em ambos os lados das fronteiras.

Dada a vantagem de abordagens em que são utilizados métodos de semidiscretização, como mostrado em Zha et al. (2019), e desenvolvendo uma nova abordagem frente ao problema de descontinuidade presente na interface, por intermédio de uma formulação em domínio único, como os que são aplicados em problemas de transferência de calor e massa (HIRATA et al., 2007; KNUPP et al., 2014; LISBOA et al., 2018; COTTA et al., 2020a; COTTA et al., 2020b; ALMEIDA et al., 2020 ), o objetivo deste trabalho é desenvolver uma formulação em domínio único, propondo uma função de transição na interface, além disso, será usado o método das linhas (SCHIERSSER; GRIFFITHS, 2008), juntamente com um esquema de volumes finitos, para obtenção de um sistema de equações diferenciais ordinárias (EDOs).
Resultados numéricos foram gerados para teor de água $(\theta)$ e pressão hidráulica $(\Psi)$, mostrando a frente de umedecimento ao longo do perfil de solo em duas camadas, em momentos diferentes, e os efeitos da condutividade hidráulica saturada quando variam ao longo do perfil do solo.

O presente artigo possui várias contribuições à literatura desenvolvida ao longo dos últimos anos, dentre eles pode-se destacar, a aplicação do método das linhas, empregando um esquema de volumes finitos (CELIA et al.,1990; MÄNNICH, 2008). Entretanto, a principal contribuição é a formalização da abordagem em domínio único, em que é proposto uma equação de transição na interface, diferente dos trabalhos anteriores que tratavam as camadas em domínios separados. Dessa forma, o presente trabalho mostra que a estratégia utilizada é uma alternativa viável ao tratamento da descontinuidade na interface.

\section{FORMULAÇÃO MATEMÁTICA}

A equação de Richards na forma- $\theta$ que governa o escoamento vertical, unidimensional transiente em solos não saturados, é dada como (GARDNER, 1958; MATTHEWS et al., 2004; ZHA et al., 2013; BRODBRIDGE et al., 2017; ZHA et al., 2019):

$$
\frac{\partial}{\partial t}=\frac{\partial}{\partial z}\left[D() \frac{\partial}{\partial z}\right] \frac{\partial K()}{\partial z}
$$

em que $z$ [L] é a coordenada vertical, definida como positiva para baixo, $K[\mathrm{~L} / \mathrm{T}]$ é a condutividade hidráulica não saturada que está em função da umidade $\theta(z, t)\left[\mathrm{L}^{3} / \mathrm{L}^{3}\right], D\left[\mathrm{~L}^{2} / \mathrm{T}\right]$ é a difusividade hidráulica da água no solo, a qual segue a relação $D=K / C, C=\mathrm{d} \theta / \mathrm{d} \psi$ é a capacidade de água no solo, $\psi[\mathrm{L}]$ é o potencial capilar, também chamada de pressão hidráulica e $t$ [T] representa o tempo.

O escoamento governado pela Equação (1) é resolvido sobre efeito de um fluxo constante na superfície superior $\left(q_{s}\right)$, e na fronteira inferior é estabelecida uma condição de saturação. A condição inicial é a distribuição do perfil de umidade que é correspondente à solução estacionária da Equação (1). Estas condições são expressas na forma- $\theta$ da seguinte forma:

$$
\begin{aligned}
& (z, 0)={ }_{0}(z), 0 \quad z \quad L \\
& \frac{\partial(0, t)}{\partial z}=\frac{K[(0, t)] q_{s}}{D[(0, t)]}, t>0 \\
& (L, t)={ }_{s}, t>0
\end{aligned}
$$

A dependência da condutividade hidráulica e da umidade com relação ao potencial capilar são dadas pela relação proposta por Gardner (1958):

$$
\begin{aligned}
K & =K_{s} e \\
& ={ }_{r}+\left(\begin{array}{rr}
s & r
\end{array}\right) e
\end{aligned}
$$




$$
C=\frac{d}{d}=\left(\begin{array}{ll}
s & r
\end{array}\right) e
$$

sendo $K_{\mathrm{s}}[\mathrm{L} / \mathrm{T}]$ a condutividade hidráulica saturada, $\theta_{\mathrm{s}}\left[\mathrm{L}^{3} / \mathrm{L}^{3}\right]$ a umidade de saturação, o valor atingido pela umidade quando todos os poros ficam cheios de água, $\theta_{r}\left[\mathrm{~L}^{3} / \mathrm{L}^{3}\right]$ a umidade residual, a quantidade de água que permanece no solo após qualquer drenagem imposta quando as forças gravitacionais cessam e $\alpha$ [1/L] um parâmetro do solo que avalia a pressão de entrada de ar no mesmo e representa a taxa de redução da condutividade hidráulica e da umidade para $\psi$ cada vez menor (SRISVASTAVA; YEH, 1991, MÄNNICH, 2008).

\subsection{Formulação de domínio único}

O modelo empregado no presente trabalho segue o problema físico proposto por Srivastava e Yeh (1991), o qual obteve uma solução analítica da equação de Richards linearizada, para infiltração vertical à taxa constate em direção ao lençol freático em solos homogêneos de duas camadas. Tal solução é frequentemente utilizada para verificar a acurácia de modelos numéricos para o escoamento insaturado em camadas relativamente muito secas. Para tal análise, os métodos numéricos sofrem com problemas de convergência e de balanço de massa (ROMANO et al., 1998; BRUNONE et al., 2003; ZHAN e NG, 2004; ZHA et al., 2013).

0 modelo físico do escoamento de água em meio poroso é representado pela Figura 1, no qual um perfil retangular de solo é adotado. $O$ material poroso tem comprimento $L$, com $N$ camadas $L_{1}, L_{2}, \ldots, L_{N}$, nas quais as cavidades estão parcialmente ocupadas por ar.

O parâmetro $\alpha$ varia entre uma camada e outra, ou seja, $\alpha_{1} \neq \alpha_{2}$, enquanto a condutividade hidráulica saturada $K_{s}$ varia em relação à posição e será tratada como uma função, na estratégia da abordagem proposta para tratar a descontinuidade presente na interface entre camadas. Nessa situação, o fenômeno de histerese é desprezado. 0 fluxo de entrada será constante e unidirecional em $z$ (positiva para baixo) e $z_{n}$, com $n=1,2, \ldots, N-1$, identifica o término de cada camada.

Figura 1 - Perfil de solo em camadas

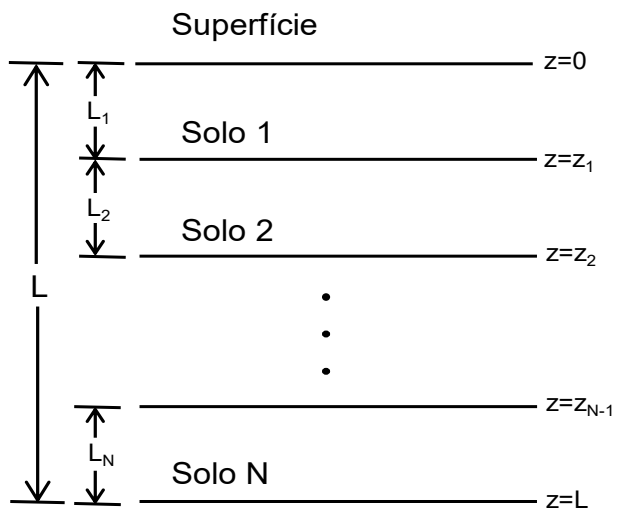

A lei de Darcy é válida para o escoamento de água líquida em meios insaturados para uma forma modificada em que a condutividade hidráulica $K$ é função do teor de umidade $\theta$, para todo domínio, descrita no trabalho de Richards (1931). A hipótese de resolver o modelo em que se apresentam duas ou mais regiões de domínio, em um único domínio, consiste em combinar as equações governantes para duas regiões em um único conjunto de equações válidas para todo o domínio. Para tal, a proposição de uma função $K_{s}(z)$ como sendo uma função de transição localizada na interface entre camadas, esta função pode ser usada para $N$ camadas. Portanto, pode-se expressar da seguinte forma:

$$
\begin{aligned}
& K_{s}(z)=\left(\begin{array}{ll}
K_{s(n)} & K_{s(n 1)}
\end{array}\right) f(z)+K_{s(n-1)}, \quad n=2,3, \ldots, N \\
& f(z)=\frac{1}{1+e^{\left[\frac{z z_{(n 1)}}{z_{(n 11)}}\right]}, \quad n=2,3, \ldots, N}
\end{aligned}
$$

em que $n$ representa a camada, $K_{s}$ a condutividade hidráulica saturada, $f(z)$ o coeficiente de transição e $\omega$ é um parâmetro de contração da faixa de transição de $K_{s}$. 0 termo $\delta=\omega \Delta z$ representa a distância antes e depois da interface entre os dois meios, no qual será realizada a mudança na propriedade $K_{s}$. 0 tamanho " $2 \delta$ " representa a faixa de transição de $K_{s}$ entre os meios e $\Delta z$ será adotado como o tamanho da malha de discretização. Assim, pode-se desenvolver uma solução em regime permanente da Equação (1) em domínio único.

\subsection{Solução para a condição inicial}

O desenvolvimento de uma solução analítica para infiltração transiente unidimensional em um perfil de solo de duas camadas foi proposto por Richards [6] e usada na solução apresentada por (MATTHEWS et al., 2004, 2005). Essa solução é válida para um caso particular em que é usado o modelo de Gardner (1958) descrito pelas Equações (5-7). Portanto, as funções hidráulicas para a forma- $\theta$ são dadas por:

$$
\begin{aligned}
& K=K_{s}\left(\frac{r}{s}\right) \\
& D=\frac{K}{C}=\frac{K_{s}}{\left(\begin{array}{lr}
s & r
\end{array}\right)}
\end{aligned}
$$

Assume-se que o parâmetro $\alpha$ é equivalente entre os tipos de solos e que para $\psi=0$, a Equação (7) resulta em $C=\alpha\left(\theta_{\mathrm{s}}-\right.$ $\left.\theta_{r}\right)$, uma constante diferente de zero. A presente solução para condição inicial fornece um meio para verificar se as condições de contorno na interface são tratadas corretamente.

Para uma infiltração vertical constante $\left(q_{l}\right)$, em direção ao lençol freático em solos homogêneos de múltiplas camadas, temse para a condição inicial em $t=0$, um perfil estacionário. Portanto, a partir da Equação (1), para um domínio único, tem-se que $\theta_{0}(z)$ pode ser obtida como: 


$$
{ }_{0}(z)={ }_{r}+\left({ }_{s}{ }_{r}\right) e^{(z L)}\left[1+q_{l} e^{L^{L}} \frac{e_{z}^{z^{\prime}}}{K_{s}\left(z^{\prime}\right)} d z^{\prime}\right], \quad 0 \quad \begin{array}{llll}
0 & z & L
\end{array}
$$

a equação obtida para $\theta_{0}(z)$ é uma solução de domínio único no qual pode ser generalizada para $N$ camadas do solo e é semi analítica pois a parte integral será resolvida por um integrador numérico. A função de transição $K_{s}(z)$ é dada pela Equação (8). A condição inicial em termos de $\psi$ pode ser obtida a partir da Equação (12) usando a relação dada na Equação (6).

\subsection{Solução numérica: Método das Linhas (Method of Lines - MOL)}

A EDP dada pela Equação (1) faz parte da classe das equações diferenciais parabólicas e é uma equação de difusão-advecção, onde o primeiro termo do lado direito representa o termo difusivo e o segundo o termo convectivo (BERARDI et al., 2017, 2018).

Pode-se encontrar na literatura diversas abordagens que tratam dos métodos numéricos para a solução da equação de Richards, os quais, na maioria, utilizam técnicas de diferenças finitas (FARTHING; OGDEN, 2017; ZHA et al., 2019). Métodos de elementos finitos ou método de volumes finitos são a base de alguns códigos populares para resolver a equação de Richards, dentre eles pode-se citar HYDRUS, RichardsFOAM e WASH123D que possuem excelentes vantagens para se resolver problemas em 2D e 3D (SIMUNEK et al., 2006; YEH; CHENG, 2011; ORGOGOZO et al., 2014).

A abordagem utilizada no presente trabalho é o método das linhas (MOL). Em geral o MOL é usado para solução de equações diferenciais parciais (EDPs) dependentes do tempo. Consiste em substituir as derivadas espaciais da EDP por aproximações algébricas, tomando as derivadas espaciais não mais declaradas explicitamente em termos de variáveis independentes espaciais. Isto explica a primeira vantagem atrativa do método das linhas. Assim, apenas a variável tempo permanece no problema físico, resultando em um sistema de equações diferenciais ordinárias (EDOs) que podem ser integradas por qualquer integrador numérico convencional (SCHIERSSER; GRIFFITHS, 2008; ÖZISIK et al., 2017).

A primeira vantagem atrativa do método das linhas, isto é, transformar um a EDP em um sistema de EDOs; Então, a conveniência de usar a abordagem MOL é que a precisão temporal pode ser especificada pelo usuário e, portanto, os recursos de adaptabilidade de intervalo de tempo disponíveis em solucionadores sofisticados de EDOs (MATTHEWS et al., 2004; BERARDI et al., 2017, 2018).

A integração da equação de Richards agora como um sistema de EDOs, é realizada em domínio único, por meio da introdução de uma função de transição na interface entre as camadas, Equações (8-9), respeitando o princípio de conservação de massa usado para desenvolver as condições de contorno na interface, como visto em (MATTHEWS et al., 2004).

\subsection{Discretização espacial: Método dos Volumes Finitos}

A discretização da Equação (1) pelo método dos volumes finitos é realizada por meio da integração em volumes de controles elementares e interpolação. 0 resultado é uma equação algébrica (MÄNNICH, 2008).

A Equação (1) foi discretizada em volumes finitos seguindo os passos descritos em (MALISKA, 2004; VERSTEEG; MALALASEKERA, 2007). O valor da variável que entra no fluxo difusivo ocorre na direção $z$, i.e., positiva para baixo, o perfil de solo é dividido em pequenos volumes na mesma direção em que é considerado o fluxo. A interpolação do termo difusivo foi aproximada pelo método ponderado a montante. A variável axial do fluxo difusivo foi dividida em $N$ intervalos, como mostrado na Figura 2. Em que $N_{z}=N_{1}+N_{2}+\ldots+N_{N}$, ou seja, $N_{1}=L_{1} / \Delta z_{1}$, $N_{2}=L_{2} / \Delta z_{2}, \ldots, N_{N}=L_{N} / \Delta z_{N}$. Esse procedimento é válido para os nós $1 \leq z \leq N_{z}-1$. A superfície do solo está em $z=0$, onde é localizado o nó $i=0$, a parte inferior da coluna do solo $z=L$ está localizado no nó $i=N_{z}$. Como se trata de um domínio único, a transição se dará em $z=z_{1}$, onde solo 1 muda instantaneamente para o solo 2 . 0 ponto $i=0$ é calculado por uma função implícita de $\theta_{0}$, dada pela Equação (12).

Figura 2 - Esquema de discretização do domínio

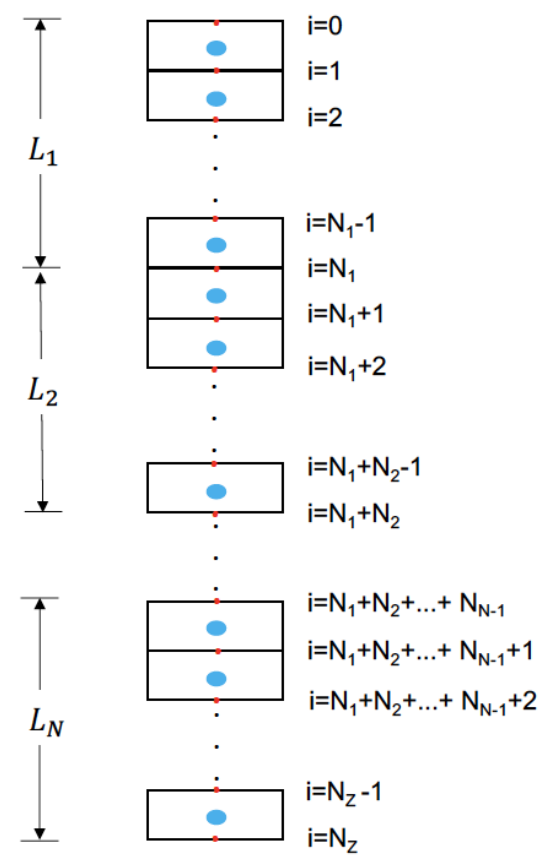

A integração deve ser realizada em cada termo da Equação (1), aplicando-se os limites do domínio da região a ser analisada. Neste caso, a aplicação dos limites de integração será apenas na variável espacial, dessa forma obtém-se a seguinte expressão:

$\int_{w}^{e} \frac{\partial}{\partial t} d z=\int_{w}^{e} \frac{\partial}{\partial z}\left(D \frac{\partial}{\partial z}\right) d z \int_{w}^{e} \frac{\partial K}{\partial z} d z$

Na presente análise, será usada como uma função de interpolação o método ponderado a montante, com a aplicação do esquema Weighted Upstream (WUDS). Dessa forma, utilizan- 
do-se o esquema de diferenças centrais, e o método de interpolação acima referenciado, obtém-se o seguinte sistema de EDOs:

$$
\begin{aligned}
& \frac{d i}{d t}=\frac{i}{z}, 1 \quad i \quad N_{z}, t>0 \\
& i=0={ }_{0}(z=0, t=0), 0 \quad i \quad N_{z} \\
& D\left({ }_{i}\right) \frac{i+1 \quad i}{z / 2}=K\left(\begin{array}{l}
i \\
i
\end{array}\right) q_{s}, t>0,1 \quad i \quad N_{z} \\
& N_{z}={ }_{s}, t>0, z=L
\end{aligned}
$$

em que,

$$
\begin{aligned}
& i_{i}=D\left({ }_{e}\right) \frac{21^{\prime}}{z}\left[\begin{array}{lll}
K\left({ }_{w}\right) & q_{s}
\end{array}\right]_{w(i=0)} \\
& =K\left({ }_{e}\right) \quad K\left({ }_{w}\right),{ }_{w}={ }_{0} \\
& { }_{0}={ }_{1} \frac{z}{2}\left[\frac{K\left({ }_{0}\right) q_{s}}{D\left({ }_{0}\right)}\right]
\end{aligned}
$$

A classificação dos nós (Figura 2) é tal que a superfície do solo $z=0$ está localizada no nó $i=0$, e a parte inferior da coluna do solo $z=L$ está localizada no nó $i=N z$. A interface entre os solos 1 e 2 está localizada no nó $z_{1}$, no pressuposto que o solo 1 muda abruptamente para o solo 2 . Observa-se que o sistema de malha é projetado para que um nó se encontre no limite da interface, a fim de se calcular as propriedades nas interfaces em termos de dois solos adjacentes. Para esse propósito, é desenvolvido uma malha em domínio único mediante a utilização de uma função de transição como função da posição z. Dessa forma, a aplicação do método das linhas leva a um único conjunto de EDOs acopladas.

\section{RESULTADOS E DISCUSSÃO}

Para avaliação do método de solução empregado (MOL), bem como o desempenho computacional da abordagem proposta no presente trabalho, foi realizada a comparação entre os resultados obtidos e a solução analítica proposta por Srivastava e Yeh (1991). A simulação foi realizada utilizando os dados referentes de quatro tipos de perfis de solos teóricos proposta pelos autores. Na Tabela 1 são apresentados os dados de entrada para os perfis de solos homogêneos com duas camadas.
Tabela 1 - Parâmetros hidráulicos de solo

\begin{tabular}{cccc}
\hline & $\alpha\left(\mathrm{cm}^{-1}\right)$ & $\mathrm{K}_{\mathbf{s} 1}(\mathrm{~cm} / \mathrm{h})$ & $\mathrm{K}_{\mathrm{s} 2}(\mathrm{~cm} / \mathrm{h})$ \\
\hline Caso 1 & 0,1 & 10 & 1 \\
Caso 2 & 0,1 & 1 & 10 \\
Caso 3 & 0,01 & 10 & 1 \\
Caso 4 & 0,01 & 1 & 10 \\
\hline
\end{tabular}

Foram adotados os seguintes valores dos parâmetros hidráulicos, $\theta_{s}=0,4, \theta_{r}=0,06, L_{1}=L_{2}=100 \mathrm{~cm}$ (altura da coluna de solo), $\theta(L, t)=0,4$ (lençol freático com $z=200 \mathrm{~cm}$ ), $q_{l}=0,1$ $\mathrm{cm} / \mathrm{h}$ (para $t=0$, regime estacionário), $q_{\mathrm{s}}=0,9 \mathrm{~cm} / \mathrm{h}$ (fluxo de entrada constante, para $t>0$ ). A duração da simulação foi de $100 \mathrm{~h}$, tempo para o qual a solução alcançou o estado estacionário. Os resultados foram obtidos em função de $\theta \mathrm{e}$, para obtenção dos resultados em função de $\psi$, a relação dada pela Equação (6) foi usada. O código foi desenvolvido em linguagem de programação FORTRAN 90/95. Para a solução do problema de valor inicial dado pelas Equações (14-20), utilizouse a sub-rotina IVPAG (IMSL, 1994) com um erro relativo de $10^{-8}$

Para o Caso 1, uma análise do comportamento da convergência de malha foi realizada, a qual é apresentada na Tabela 2. Os resultados foram obtidos para o potencial $\theta$ em diferentes posições e diferentes pontos na malha, $N_{z}$. Conforme mostrado na Tabela 2, os valores de $\theta$ foram mostrados em diferentes posições ao longo do perfil e avaliados em dois tempos, para $t=1 \mathrm{~h}$ e $100 \mathrm{~h}$, com o objetivo de se verificar uma possível degeneração do algoritmo computacional ao longo do tempo e verificar a convergência numérica para o conteúdo de água $\theta$, ao longo das camadas de infiltração. Observa-se que os valores de $\theta$ apresentam boa convergência com $N_{z}=400$ para as posições $z=10 \mathrm{~cm} \mathrm{e} z=190 \mathrm{~cm}$ próximos à superfície e ao lençol freático, respectivamente. Para as posições internas, próximas à interface, na presença de uma barreira capilar, e da descontinuidade, é observado que à medida que se aumenta o número de pontos da malha, os valores tendem a convergir em três e cinco dígitos significativos, o que atesta a qualidade do código e dos resultados obtidos.

Foi realizada uma análise para se verificar a influência do parâmetro de contração $\omega$ do coeficiente de transição. A Figura 3 apresenta a influência do parâmetro $\omega$ na solução do problema em termos da pressão hidráulica em função da profundidade. Nesta figura foi plotado a pressão hidráulica para diferentes valores do parâmetro de contração da faixa de transição de Ks (i.e., $\omega=0.3,0.5,1.0$ e 10) e, também, se comparou com os resultados de Srivastava e Yeh (1991) para o Caso 1 , no tempo igual a $1 \mathrm{~h}$. 
Tabela 2 - Análise de convergência para $\mathrm{t}=1 \mathrm{~h}$ e $\mathrm{t}=100 \mathrm{~h}$

\begin{tabular}{|c|c|c|c|c|c|}
\hline \multicolumn{6}{|c|}{$t=1 h$} \\
\hline$N_{z}$ & $z=10 \mathrm{~cm}$ & $z=95 \mathrm{~cm}$ & $z=100 \mathrm{~cm}$ & $z=105 \mathrm{~cm}$ & $z=190 \mathrm{~cm}$ \\
\hline 50 & 0,0849 & 0,078 & 0,090 & 0,093 & 0,202 \\
\hline 100 & 0,0849 & 0,079 & 0,092 & 0,097 & 0,204 \\
\hline 200 & 0,0849 & 0,080 & 0,093 & 0,097 & 0,205 \\
\hline 400 & 0,0849 & 0,081 & 0,092 & 0,095 & 0,206 \\
\hline \multicolumn{6}{|c|}{$t=100 h$} \\
\hline$N_{z}$ & $z=10 \mathrm{~cm}$ & $z=95 \mathrm{~cm}$ & $z=100 \mathrm{~cm}$ & $z=105 \mathrm{~cm}$ & $z=190 \mathrm{~cm}$ \\
\hline 50 & 0,0906 & 0,174 & 0,303 & 0,273 & 0,376 \\
\hline 100 & 0,0906 & 0,203 & 0,331 & 0,340 & 0,377 \\
\hline 200 & 0,0906 & 0,226 & 0,347 & 0,364 & 0,378 \\
\hline 400 & 0,0906 & 0,240 & 0,356 & 0,366 & 0,378 \\
\hline
\end{tabular}

Figura 3 - Analise do parâmetro de contração da faixa de transição de $K_{s}$, para valores de $\omega=0.3,0.5,0.1$ e 10 , e comparação com os valores de referência de Srivastava e Yeh (1991), para o Caso 1, com o tempo de $1 \mathrm{~h}$

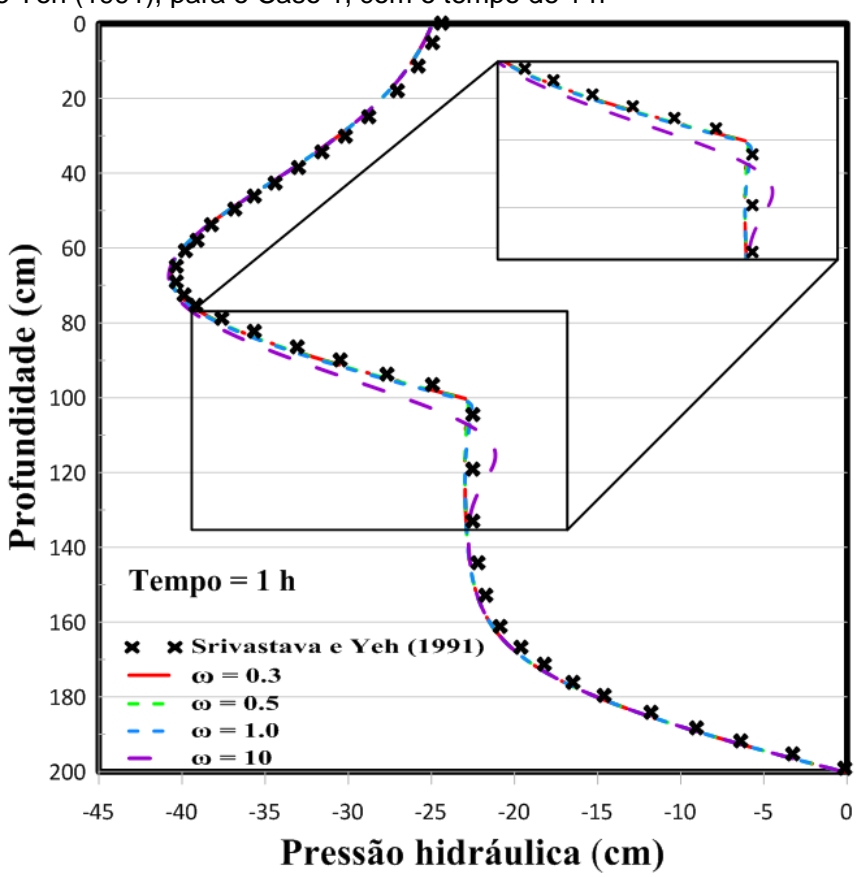

Para valores de $\omega<0.3$ a função de transição dada pela Equação (9) tende a diminuir a espessura da região de transição, aproximando-se de valores próximos a zero para $\delta$, gerando uma singularidade no modelo de transição com impacto negativo na solução numérica do modelo. Para valores de $\omega>0.3$ a singularidade da transição da propriedade $K_{s}$ é suavizada a solução do problema apresenta resultados numérica e fisicamente consistentes. Para valores $\omega$ entre 0.3 à 0.1 , não há diferença significativa nos resultados apresentados. Contudo, para proposição em que $\omega=10$, aumentando-se faixa de transição de $K_{s}$ entre os meios, observa-se um distanciamento maior entre os valores de referência e os valores calculados, próximos a interface. Isso se dá pelo aumento da espessura da região de transição, tendo como consequência problemas em preservar os valores físicos próximos a transição entre camadas. Dessa forma, optou-se por utilizar $\omega=0.3$.
Na Figura 4 são mostrados os resultados de $\theta$, em diferentes horas e a comparação dos resultados numéricos obtidos em função da pressão hidráulica $\psi$, com os resultados analíticos de Srivastava e Yeh (1991), para um valor de pontos na malha de $N_{z}=400$, e $\Delta z=0.5 \mathrm{~cm}$ e $\omega=0.3$.

Uma excelente concordância é observada entre os presentes resultados e os da solução analítica de Srivastava e Yeh (1991), o que garante a verificação dos resultados obtidos com o enfoque do método das linhas.

Buscando-se analisar o comportamento hidráulico dos solos sujeitos a uma taxa constante de infiltração durante o processo de umedecimento, observa-se diferentes padrões dos efeitos de $K_{s}$ para o Caso $1 \mathrm{em}$ que, $K_{s 1}>K_{s 2}$. Por ser uma propriedade que relaciona a condutibilidade do solo saturado, 
referente à capacidade com que a água tende a fluir pelos poros. Nota-se que a frente de umedecimento na primeira camada tende a se propagar mais rapidamente que na segunda camada, ocupando os espaços porosos vazios, e forçando um efeito de decréscimo sobre a pressão hidráulica ao longo do perfil.

Figura 4 - Distribuição do conteúdo de água e de pressão hidráulica para $\alpha=0.1 \mathrm{~cm}^{-1}, \mathrm{Ks}_{1}=10(\mathrm{~cm} / \mathrm{h}), \mathrm{Ks} 2=1(\mathrm{~cm} / \mathrm{h}), \mathrm{Nz}=400, \Delta \mathrm{z}=0.5 \mathrm{~cm}$, $\omega=0.3$ e t=1, 20 e 100 h: (a) Solução MOL para o conteúdo de água; (b) Comparação do presente resultado com os de Srivastava e Yeh (1991) para a pressão hidráulica

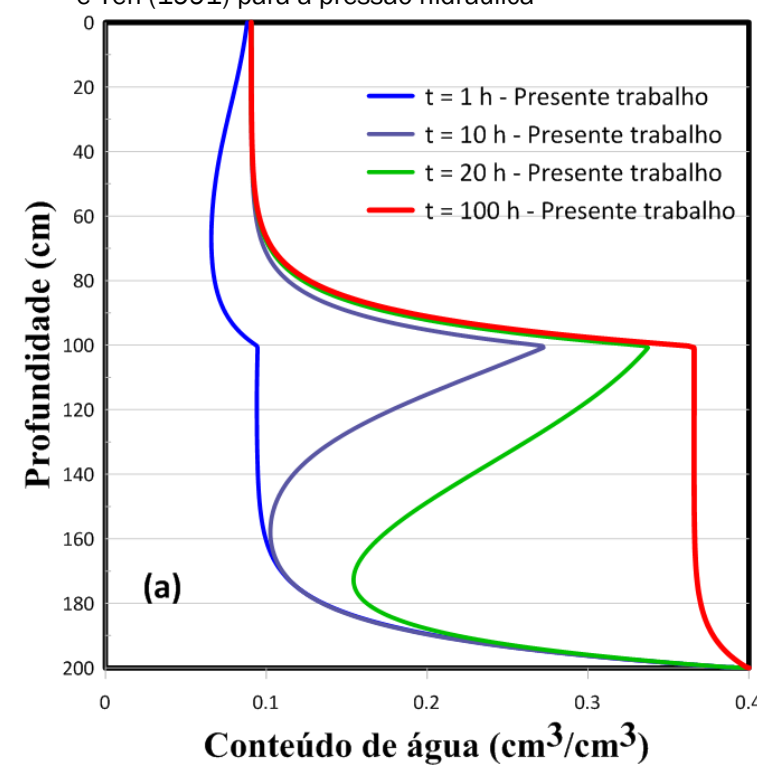

O comportamento da função $\mathrm{K}$, bem estabelecida nas referências de Richards (1931) e de Libardi (2005), tende a reduzir rapidamente quando $\theta$ diminui com seu valor de saturação. Alguns dos motivos estão relacionados com o decréscimo da seção transversal disponivel total para o fluxo à medida que $\theta$ decai, e ocupação dos poros maiores primeiramente, quando $\theta$ diminui. Na Figura 5 é apresentado o comportamento desse

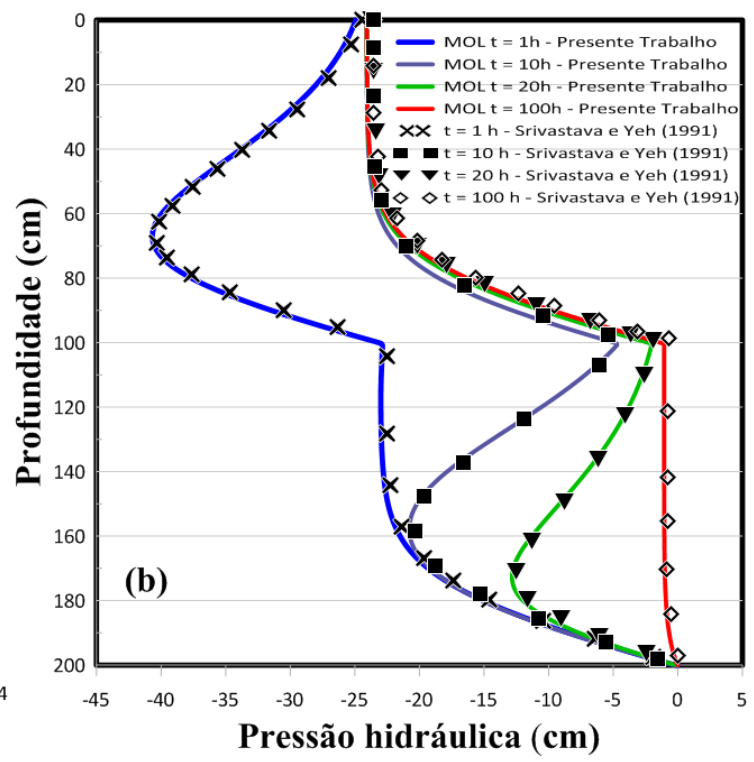

efeito para o Caso 2, o qual adotou-se um valor de $\alpha=0.1 \mathrm{~cm}$ 1 para ambos os solos. Dado agora que Ks1 < Ks2, observase a inversão dos valores de pressão de entrada, e redução da taxa da condutividade hidráulica e da umidade à medida que a pressão hidráulica é cada vez menor, observando que para Ks maiores a pressão de entrada é mais elevada.

Figura 5 - Distribuição do conteúdo de água e de pressão hidráulica para $\alpha=0.1 \mathrm{~cm}^{-1}, \mathrm{Ks}_{1}=1(\mathrm{~cm} / \mathrm{h}), \mathrm{Ks} 2=10(\mathrm{~cm} / \mathrm{h}), \mathrm{Nz}=400, \Delta \mathrm{z}=0.5 \mathrm{~cm}$, $\omega=0.3$ e t=1, 20 e 100 h: (a) Solução MOL para o conteúdo de água; (b) Comparação do presente resultado com os de Srivastava e Yeh (1991) para a pressão hidráulica

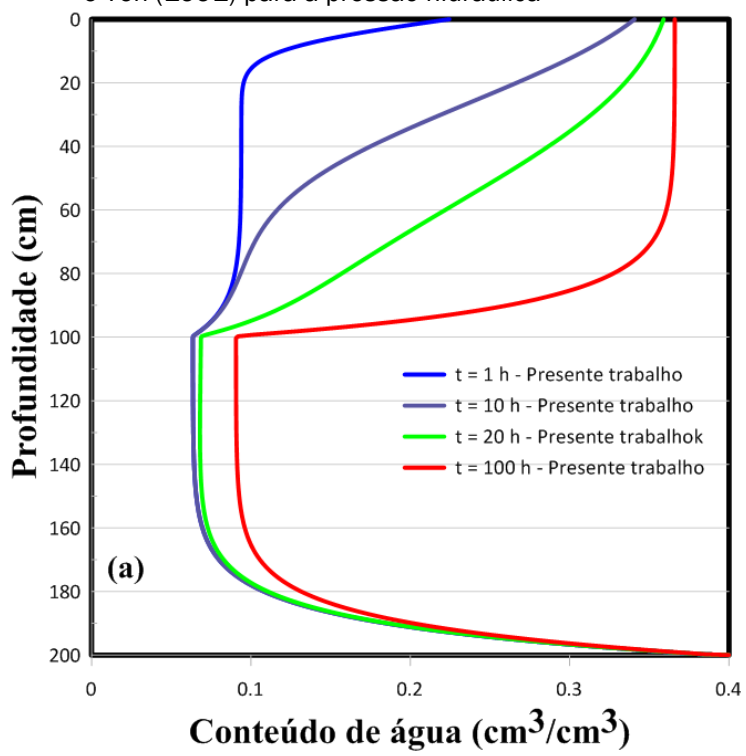

Em seguida a simulação ocorre para valores de $\alpha$ menores, com o intuído de avaliar seus efeitos sobre os comportamentos das curvas de umedecimento e de pressão hidráulica. As

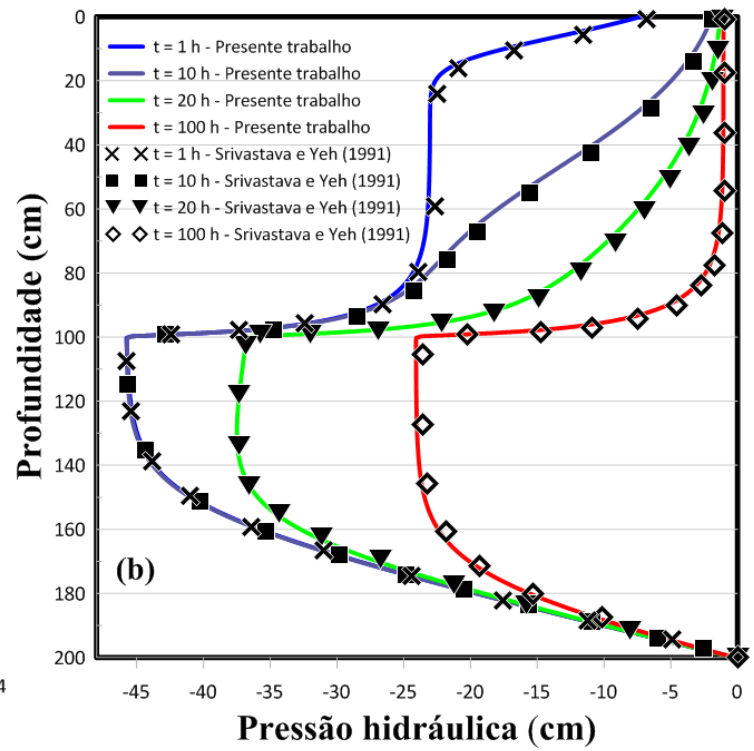

Figuras 6 e 7 apresentam os resultados dos Casos 3 e 4 para $\alpha=0.01 \mathrm{~cm}-1$. Quando Ks1 > Ks2 (Figura 6), observa-se o aumento da velocidade com que a frente de umedecimento 
ocorre, quando comparada ao valor de $\alpha$ anterior. Pode-se verificar que para menores valores de $\alpha$, maior será a pressão de entrada de ar no solo, reduzindo a taxa de condutividade hidráulica e da umidade à medida que a pressão hidráulica diminui, e que $\alpha$ permanece constante, para os ciclos de umedecimento e drenagem, quando o fenômeno de histerese é desprezível.

Para o Caso 4 em que Ks1 < Ks2, além da pressão de entrada diminuir, nota-se que as curvas de umedecimento são mais dispersas (Figura 7a) quando Ks menor. Isso ocorre principalmente pela pressão de entrada menor, como pode ser visto na Figura $7 b$, e se repete para os outros casos, uma maior dispersão das curvas de umedecimento quando a pressão de entrada da primeira camada é menor que na segunda. Portanto, para valores de $\alpha$ menores e Ks maiores, a velocidade de propagação será maior.

Quanto à descontinuidade presente na interface entre as camadas, essa é fisicamente explicada pela mudança das propriedades hidráulicas da camada 1 para camada 2 . Nota-se que a pressão hidráulica de entrada na interface é mais elevada para os valores de Ks maiores, e para os valores $\alpha$ menores. Esse efeito se reflete na velocidade com que o escoamento alcança o regime estacionário.

Figura 6 - Distribuição do conteúdo de água e de pressão hidráulica para $\alpha=0.01 \mathrm{~cm}^{-1}, \mathrm{Ks}_{1}=10(\mathrm{~cm} / \mathrm{h}), \mathrm{Ks}_{2}=1(\mathrm{~cm} / \mathrm{h}), \mathrm{Nz}=400, \Delta \mathrm{z}=0.5 \mathrm{~cm}$, $\omega=0.3$ e t=1, 20 e 100 h: (a) Solução MOL para o conteúdo de água; (b) Comparação do presente resultado com os de Srivastava e Yeh (1991) para a pressão hidráulica
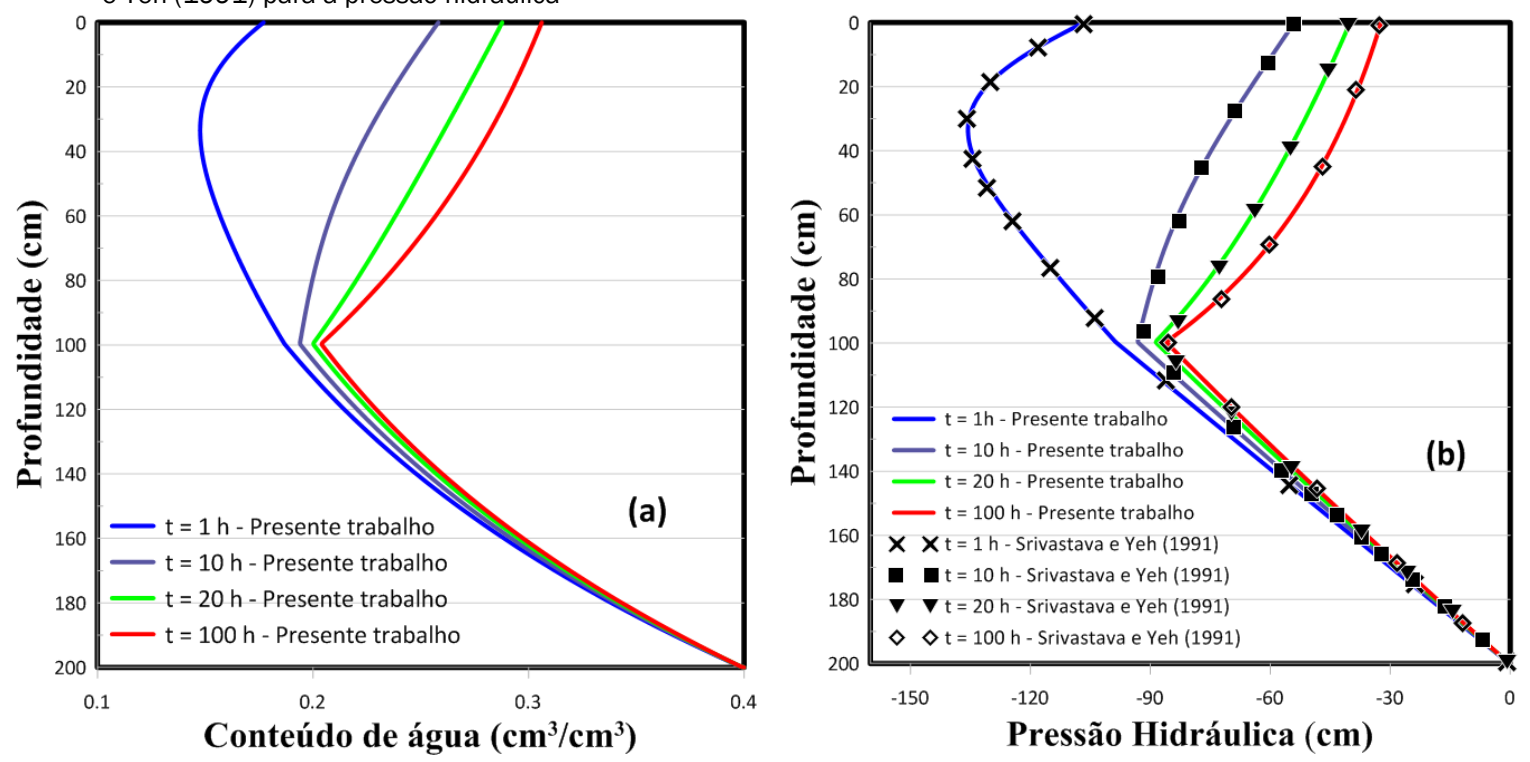

Figura 7 - Distribuição do conteúdo de água e de pressão hidráulica para $\alpha=0.01 \mathrm{~cm}^{-1}, \mathrm{Ks}_{1}=1(\mathrm{~cm} / \mathrm{h}), \mathrm{Ks} \mathrm{s}_{2}=10(\mathrm{~cm} / \mathrm{h}), \mathrm{Nz}=400, \Delta \mathrm{z}=0.5 \mathrm{~cm}$ $\omega=0.3$ e t=1, 20 e 100 h: (a) Solução MOL para o conteúdo de água; (b) Comparação do presente resultado com os de Srivastava e Yeh (1991) para a pressão hidráulica
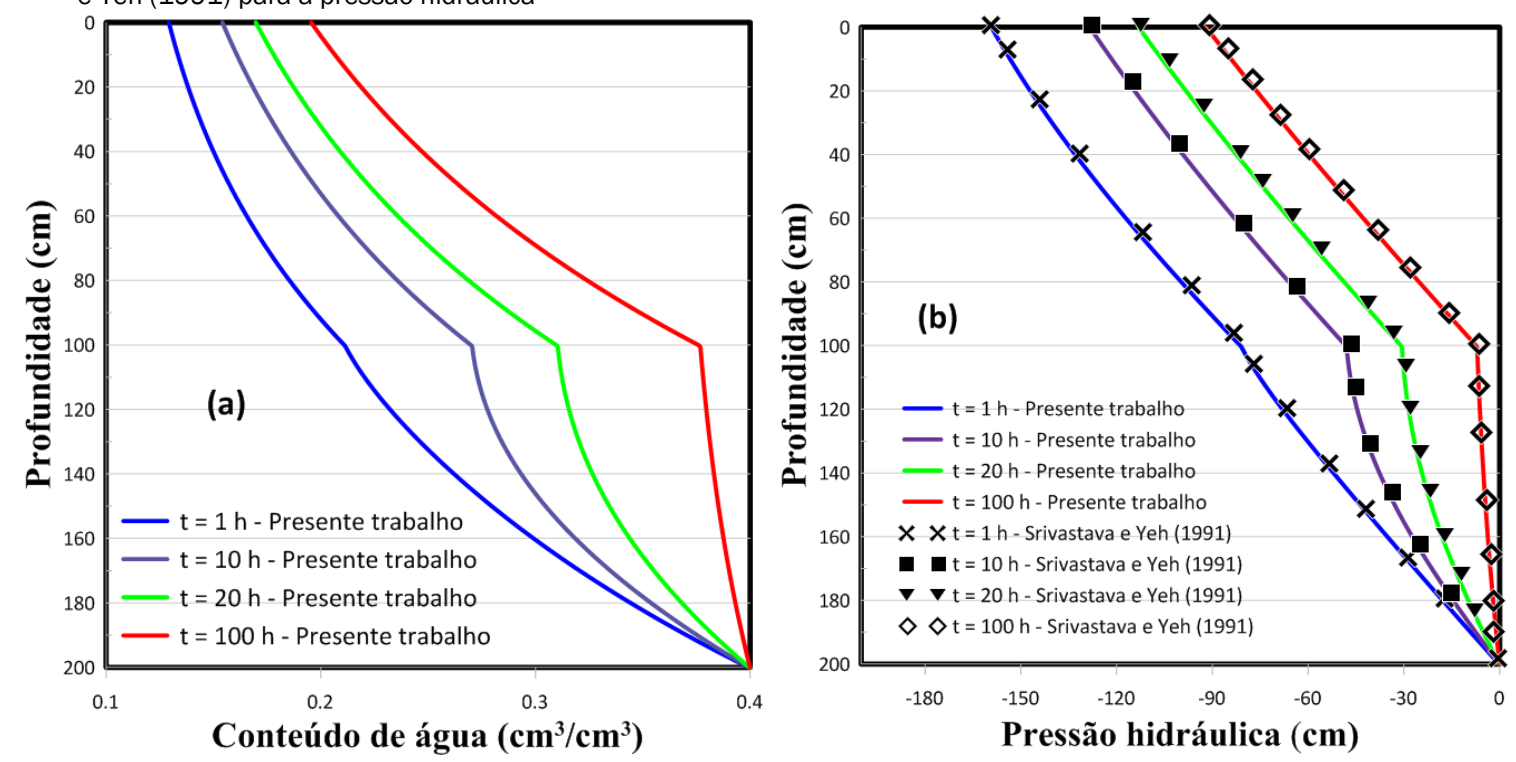
A comparação entre a solução numérica e a solução analítica melhoram nossa compreensão do processo de infiltração sob o estado transiente em solos estratificados. E, embora as soluções baseadas nas relações funcionais exponenciais sejam restritas para quaisquer aplicações práticas, elas servem como meios para verificar muitos modelos numéricos; para escoamentos insaturados, especialmente para infiltração de solos em camadas muito secos onde os modelos numéricos frequentemente sofrem de convergência e problemas de equilíbrio de massa.

Dessa forma, o objetivo principal do presente trabalho, é a proposição de uma função de transição para tratar a descontinuidade presente na interface entre camadas, em que a modelagem em domínio único só é possível pelo uso da Equações (89), como função de transição. Essas podem ser generalizadas para $\mathrm{N}$ camadas. A análise agora mostrada na Figura 8 considera 4 camadas com $\mathrm{L} 1=\mathrm{L} 2=\mathrm{L} 3=\mathrm{L} 4=100 \mathrm{~cm}, \mathrm{Ks} 1=10 \mathrm{~cm} / \mathrm{h}$, $\mathrm{Ks} 2=7 \mathrm{~cm} / \mathrm{h}, \mathrm{Ks} 3=4 \mathrm{~cm} / \mathrm{h}, \mathrm{Ks} 4=1 \mathrm{~cm} / \mathrm{h}, \alpha=0.1$ e todos os outros parâmetros hidráulicos anteriores mantidos constan- tes. As condições iniciais seguem também os casos anteriores, assim como a homogeneidade do perfil de solo a ser analisado. Dessa forma, a infiltração de água no solo agora se dá na presença de mais de duas camadas, em que a condutividade hidráulica saturada decresce com a profundidade, ou seja, Ks1 > Ks2 > Ks3 > Ks4, até o lençol freático, dado que novamente os efeitos de histerese são desprezados, e que $\alpha$ é constante, ao longo de todo o perfil. E assim como nos outros casos, observa-se que o comportamento dos perfis de umedecimento e de pressão hidráulica são semelhantes aos casos anteriores. O conteúdo de água é crescente quando se compara as camadas superiores com as inferiores, bem como a pressão hidráulica, a qual representa a pressão de entrada sobre a camada, e que é proporcional à medida que Ks decai. Os resultados obtidos demonstram que por intermédio de soluções numéricas pode-se compreender o fenômeno de infiltração em perfis de solo em multicamadas, e que podem auxiliar em modelos práticos, e em experimentos de campo, contribuindo para cada vez mais se busque resultados mais próximos dos modelos reais.

Figura 8 - Distribuição de conteúdo de água e de pressão hidráulica para $\alpha=0.1 \mathrm{~cm}^{-1}, \mathrm{Ks}_{1}=10(\mathrm{~cm} / \mathrm{h}), \mathrm{Ks} \mathrm{s}_{2}=7(\mathrm{~cm} / \mathrm{h}), \mathrm{Ks}_{3}=4(\mathrm{~cm} / \mathrm{h})$, $\mathrm{Ks}_{4}=1(\mathrm{~cm} / \mathrm{h}), \mathrm{Nz}=400, \Delta \mathrm{z}=0.5 \mathrm{~cm}, \omega=0.3$, e t=1, 20 e $100 \mathrm{~h}$ : (a) Solução MOL para o conteúdo de água; (b) Solução MOL para a pressão hidráulica

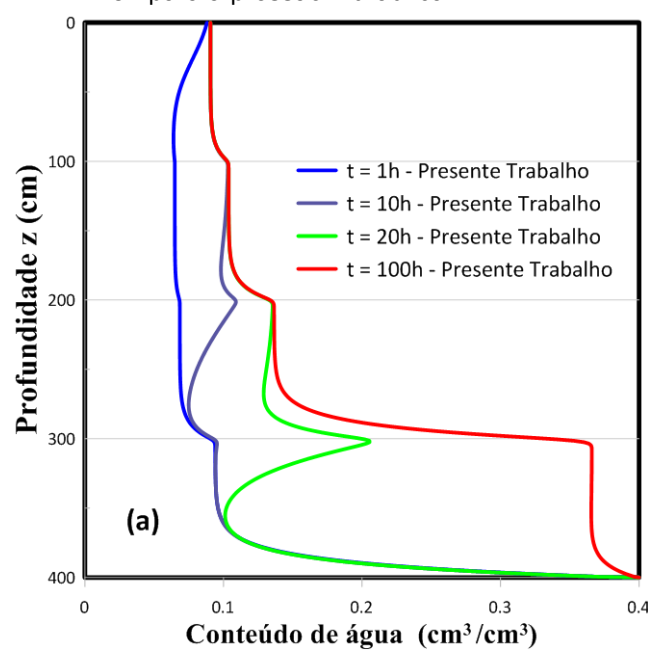

\section{CONCLUSÃO}

O presente estudo teve como contribuição fundamental o desenvolvimento de uma técnica de solução em domínio único, e a proposição de uma função de transição para que isso fosse possivel. Os resultados numéricos, por sua vez, foram verificados por meio de uma solução analítica para o modelo em questão, e como tal é importante para compreensão do fenômeno de infiltração de água em solos estratificados, homogêneos, bem como o entendimento da importância de alguns parâmetros hidráulicos para o fenômeno de infiltração, e para o planejamento experimental de futuros trabalhos.

Os resultados obtidos aqui, fundamentam os esforços para desenvolver modelos cada vez mais realísticos, e que possam cada vez mais contribuir com o entendimento dos fenômenos físicos que envolvem os processos de infiltração e fenômenos

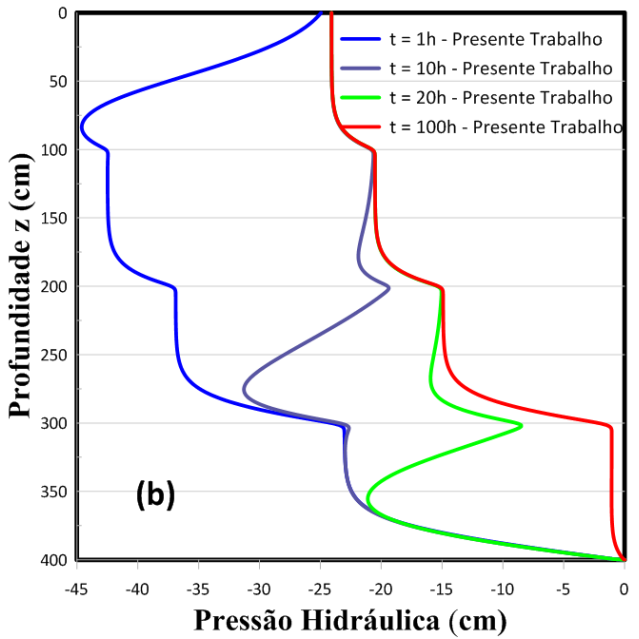

correlatos, além disso, mostram que a técnica utilizada no presente trabalho é uma ferramenta robusta para a abordagem do modelo adotado no presente trabalho.

\section{AGRADECIMENTOS}

Os autores gostariam de agradecer à Coordenação de Aperfeiçoamento de Pessoal de Nível Superior (CAPES) pelo apoio financeiro durante o período dos estudos de M.Sc. do autor Diego Sousa Lopes.

\section{REFERÊNCIAS}

ALMEIDA, A.P.; NAVEIRA-COTTA, C.P.; COTTA, R.M. Transient Multidimensional Heat Conduction in Heterogeneous Media: Integral Transform with Single Domain Formulation. Int. Comm. Heat \& Mass Transfer, v.117, p. 104792 , 2020. https://doi.org/10.1016/i.icheatmasstransfer.2020.104792 
BERARDI, M.; DIFONZO, F.; NOTARNICOLA, F.;, VURRO, M. A transversal method of lines for the numerical modeling of vertical infiltration into the vadose zone. Applied Numerical Mathematics, v. 135, p. 264-275, 2019. https://doi.org/10.1016/i.apnum.2018.08.013

BERARDI, M.; DIFONZO, F.;VURRO, M.; LOPEZ L. The 1D Richards' equation in two layered soils: a Filippov approach to treat discontinuities. Advances in water resources, v. 115, p. 264-272, $2018 . \quad$ https://doi.org/10.1016/i.advwatres.2017.09.027

BROADBRIDGE, P.; DALY, E.; GOARD, J. Exact Solutions of the Richards Equation with Nonlinear Plant-Root Extraction. Water Resources Research, v. 53, n. 11, p. 9679-9691, 2017. https://doi.org/10.1002/2017WR021097

BRUNONE, B.; FERRANTE, M.; ROMANO, N.; SANTINI, A. Numerical simulations of one-dimensional infiltration into layered soils with the Richards equation using different estimates of the interlayer conductivity. Vadose Zone Journal, v. 2, n 2, p. 193-200, 2003. https://doi.org/10.2113/2.2.193

CELIA, A,; BOULOUTAS, T.; ZARBA, L. A general massa-conservative numerical solution for the unsaturated flow equation. Water Resources Research. v.26, n 7, p.1483-1496, 1990. https://doi.org/10.1029/WR026i007p01483

COTTA, R.M.; LISBOA. K.M.; ZOTIN, J.L.Z.; Integral transforms for flow and transport in continuum and discrete models of fractured heterogeneous porous media, Water Resources, v. 142, n. 2, p.1-17, 2020a. https://doi.org/10.1016/i.advwatres.2020.103621

COTTA, R.M.; NAVEIRA-COTTA,; C.P.; VAN GENUCHTEN, M.T.; SU, J.; QUARESMA, J.N.N.; Integral Transform Analysis of Radionuclides Migration in Variably Saturated Media with Physical Non-equilibrium Model: Application to Solid Waste Leaching in Uranium Mining. Annals of the Brazilian Academy of Sciences, v.92, n.1, p. 1-28, 2020b. https://doi.org/10.1590/0001-3765202020190427

FARTHING, M.W.; OGDEN, F.L.; Numerical Solution of Richards' Equation: A Review of Advances and Challenges. Soil Science Society of America Journal, v. 81, n. 6, p. 1257-1269, 2017. https://doi.org/10.2136/sssaj2017.02.0058

GARDNER, W. Some steady-state solutions of the unsaturated moisture flow equation with application to evaporation from a water table. Soil science, v. 85, n. 4, p. 228-232, 1958. https://doi.org/10.1097/00010694-195804000-00006

HILSS R; PORRP I; HUDSON B; WIERENGA J. Modeling one-dimensional infiltration into very dry soils 1 . Model development and evaluation. Water Resources Research, v.25, n 6, p.12591269, 1989. https://doi.org/10.1029/WR025i006p01259

HIRATA, S.; GOYEAU, B.; GOBIN, D.; NAVEIRA-COTTA, C.; COTTA, R. M. Linear stability of natural convection in superposed fluid and porous layers: Influence of the interfacial modelling. International Journal of Heat and Mass Transfer. v.50, n 7-8, p.1356-1367, 2007. https://doi.org/10.1016/i.ijheatmasstransfer.2006.09.038

IMSL Library, MATH/LIB, Houston, TX, 1994.

KANZARI, S.; MARIEM, S. Kirchhoff Transformation of Richards Equation for Simulating Water Flow in Porous Media. System
Science and Apllied Mathematics, v.2, n 2, p. 8-12, 2017.

KNUPP, D.; COTTA, R.; NAVEIRA-COTTA, C.; KAKAÇ, S. Transient conjugated heat transfer in microchannels: Integral transforms with single domain formulation. International Journal of Thermal Sciences, v. 88, p. 248-257, 2015. https://doi.org/10.1016/i.ijthermalsci.2014.04.017

LAIO, F.; PORPORATO, A.; RIDOLFI, L.; RODRIGUEZ I. Plants in water-controlled ecosystems: Active role in hydrologic processes and response to water stress. Adv. Water Resour. v. 24, n 7, p. 707-723, 2001. https://doi.org/10.1016/S03091708(01)00005-7

LIBARDI, P. L. Dinâmica da Água no Solo. v. 61. Edusp, 2005.

LISBOA, K.; SU, J.; COTTA, R. M. Single domain integral transform analysis of natural convection in cavities partially filled with heat generating porous medium. Numerical Heat Transfer, Part A: Applications. v.74, n 3, p. 1068-1086, 2018. https://doi.org/10.1080/10407782.2018.1511141

MALISKA, C. R. Transferência de calor e mecânica dos fluidos computacional. 2. ed. Rio de Janeiro: Livros Técnicos e Científicos Editora, v. 200, 2004.

MÄNNICH, M. Desenvolvimento de soluções analítica e numérica da equação de Richards. [Dissertação]. Curitiba (PR): Universidade Federal do Paraná; p.129, 2008.

MATTHEWS, C. J.; COOK, F. J.; KNIGHT, J. H.; BRADDOCK, R. D. Handling the water content discontinuity at the interface between layered soils within a numerical scheme. Soil Research, v. 43, n. 8, p. 945-955, 2006. https://doi.org/10.1071/SR05069

MATTHEWS, C.; BRADDOCK, D.; SANDER, G. Modeling Flow Through a One-Dimensional Multi-Layered Soil Profile Using the Method of Lines. Environmental Modeling \& Assessment. v. $9, \quad$ n. 2, p.103-113, 2004 https://doi.org/10.1023/B:ENMO.0000032092.10546.c6

ORGOGOZO, L.; RENON, N.; SOULAINE, C.; HÉNON, F.; TOMER, S.K,; QUINTARD, M. An open source massively parallel solver for Richards equation: Mechanistic modelling of water fluxes at the watershed scale. Computer Physics Communications, v. 185, n. 12, p. 3358-3371, 2014. https://doi.org/10.1016/j.cpc.2014.08.004

ÖZISIK, M.N.; ORLANDE H. R. B.; COLAÇO, M. J.; COTTA, R. M. Finite difference methods in heat transfer. 2. ed. New York: CRC Press. 2017. https://doi.org/10.1201/9781315168784

PHILIP J. Theory of Infiltration:1.the Infiltration Equation and Its Solution. Soil Science, v. 83, n. 5, p. 345-358, 1957.10. Hillel D. Environmental soil physics. San Diego: Academic Press; 1998.144 p. https://doi.org/10.1097/00010694195705000-00002

RICHARDS LA. Capillary conduction of liquids through porous mediums. Physics Rev. v.54, n 6, p. 318-333, 1931. https://doi.org/10.1063/1.1745010

ROMANO, N.; BRUNONE, B.; SANTINI A. Numerical analysis of one-dimensional unsaturated flow in layered soils. Advances in Water Resources. v. 21, n. 4, p. 315-324, 1998. https://doi.org/10.1016/S0309-1708(96)00059-0 
ROMANO, N.; BRUNONE, B.; SANTINI, A. Numerical analysis of one-dimensional unsaturated flow in layered soils. Advances in Water Resources. v.21, n 4, p.315-324, 1998. https://doi.org/10.1016/S0309-1708(96)00059-0

SCHIESSER, William E.; GRIFFITHS, Graham W. A compendium of partial differential equation models: method of lines analysis with Matlab. Cambridge University Press, 2009. https://doi.org/10.1017/CB09780511576270

SIMUNEK, J.; VAN GENUCHTEN, M.; SEJNA M. The HYDRUS software package for simulating the two-and three-dimensional movement of water, heat, and multiple solutes in variably saturated media. Technical manual, version, v. 1, p. 241, 2006.

SRIVASTAVA, R.; YEH, T. Analytical solutions for one-dimensional, transient infiltration toward the water table in homogeneous and layered soils. Water Resources Research, v. 27, n 5, p.753-762,1991. https://doi.org/10.1029/90WR02772

VERSTEEG, Henk Kaarle; MALALASEKERA, Weeratunge. An introduction to computational fluid dynamics: the finite volume method. Pearson education, 2007.

WENDLAND, E.; PIZARRO, M. Modelagem computacional do fluxo unidimensional de água em meio não saturado do solo. Engenharia Agrícola, Associação Brasileira de Engenharia Agrícola, v.30, $\mathrm{n} 3, \quad \mathrm{p}$ 424-434, 2010. https://doi.org/10.1590/S0100-69162010000300007
YEH, G.T.; SHIH,D.S.; CHENG, J.R.C. An integrated media, integrated processes watershed model. Computers and Fluids. Computers \& Fluids, v. 45, n. 1, p. 2-13, 2011. https://doi.org/10.1016/j.compfluid.2010.11.018

ZHA, Y.; YANG, J.; ZENG, J.; TSO, C. H.; ZENG, W.; SHI L. Review of numerical solution of Richardson-Richards equation for variably saturated flow in soils. Wiley Interdisciplinary Reviews: Water. v.6, n 5, p.1-23, 2019. https://doi.org/10.1002/wat2.1364

ZHA, Y.; YANG, J.; SHI, L.; SONG X. Simulating One-Dimensional Unsaturated Flow in Heterogeneous Soils with Water Content-Based Richards Equation. Vodose Zone Journal, v. 12, n 2, p.1-13, 2013. https://doi.org/10.2136/vzj2012.0109

ZHAN, T. L.T.; NG, C.W.W. Analytical analysis of rainfall infiltration mechanism in unsaturated soils. International Journal of Geomechanics, v. 4, n. 4, p. 273-284, 2004. https://doi.org/10.1061/(ASCE)1532-3641(2004)4:4(273)

ZHU, H.; LIU, T.; XUE, B.; YINGLAN, A.; WANG G. Modified Richrads'Equation to Improve Estimates of Soil Moisture in Two-Layered Soils after Infiltration. Water Rev, v.10, n 9, p.1174-1190, 2018. https://doi.org/10.3390/w10091174 